\title{
Rhythm Drivers, Physical Properties, Mechanisms of Formation
}

\author{
Mikhail Mazurov,** \\ ${ }^{1}$ Russian University of Economics. G.V. Plekhanov, RU-117997, Moscow, Russia
}

\begin{abstract}
A mathematical model of the pacemaker is presented in the form of a nonlinear system of ordinary differential equations and in the form of a system of partial differential equations for distributed pacemakers. For the numerical study of the properties of the pacemaker, a modified axiomatic WienerRosenbluth method was used using the properties of uniform almost periodic functions. Physical foundations, mechanisms of formation, properties of point and distributed pacemakers are described in detail.
\end{abstract}

\section{Introduction}

Rhythm generators are widely represented in physics, biology, physiology, nature, ecology and economics. The entire human body is permeated with rhythm generators. Some of the rhythm generators in the human body are shown in fig. 1 .

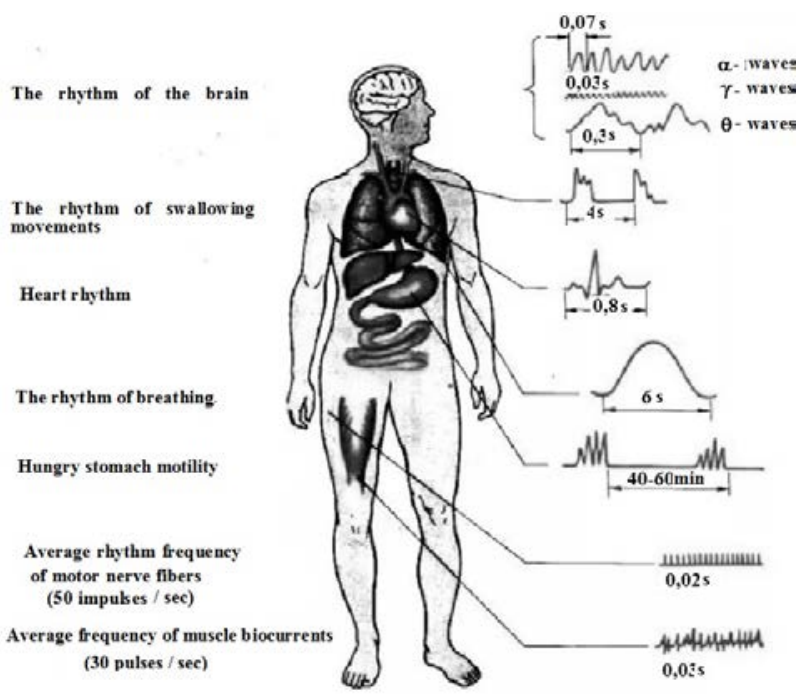

Fig. 1. Rhythm generators in the human body.

The entire human body is permeated with pacemakers. There are two types of neural networks responsible for rhythm generation. The first, using interconnected neurons, pacemakers or pacemakers, called a pacemaker (DR). The second type, a neural network based on the interaction of neuronal cells, devoid of their own pacemaker activity, but connected in a network. Such oscillators are called network or central rhythm generators (CGR). The emergence of oscillatory, rhythmic activity is due to the emergence of positive feedback in the network system. CGRs have a variety of structures and complexity.
In this work, we will consider DR based on relaxation oscillators that have their own pacemaker activity, called pacemakers; CGRs are not considered.

Let's define the physics of processes during the functioning of DR and the problems that arise during their study. These are the following problems:

1) Physical processes during the formation of a single rhythm or synchronization of partial rhythms of separate relaxation oscillators that make up DR. This is a complex problem in the physics of mutual synchronization of relaxation self-oscillations; 2) If the VR consists of several independent parts, then there is a need for mutual synchronization of individual parts. For example, the BP of respiration, located in the human medulla oblongata, consists of several parts that control various objects - the intercostal muscles, the abdominal diaphragm, which must work synchronously and must be rigidly synchronized; 3 ) The problem of synchronization of macroscopic VR with other subsystems of the body and the external environment. For example, the rhythm of the pacemaker of the heart or respiration as a whole should be determined by the internal state of the body and external load; 4) The problem of synchronization of macroscopic DR with the external environment of the organism. For example, a single DR rhythm located in the suprachiasmatic nucleus of the human medulla oblongata, which has a period of 23-25 hours, should be synchronized with a 24-hour circadian rhythm; 5) Cognitive intelligent regulation of the pacemaker depending on information from the external environment. For example, the formation of the rhythm in the hippocampus to realize the state of attention in the brain. Another striking example is the suppression of the alpha-rhythm generator in certain parts of the brain neocortex that are not involved in the processing of current essential information; 6) Real biological oscillators are a self-oscillating relaxation system (RSOS). This is due to the fact that effective mutual synchronization of the partial oscillators and components of the DR and synchronization of the DRs 
themselves with each other and with the environment is possible if these are relaxation oscillators. This is due to the fact that the synchronization band of harmonic oscillators is much smaller than the synchronization band of the RSOS. A DR composed of mutually synchronized harmonic oscillators will not be a reliable, stable macroscopic system; 7) Influence of the spatial distribution of partial oscillators. The fact is that the nearest oscillators have the greatest influence on a particular oscillator, while those located at a great distance have less influence. Thus, real DR should be viewed as a distributed active environment; 8) The formation of a single rhythm can be significantly influenced by autowave interactions in a distributed active environment; 9) DR must transmit a stable signal of sufficient electrical power to the environment; Currently, the mechanisms of the formation of a single rhythm in the heart have been studied to a greater extent, this problem has been more than a century. Approaches to understanding the formation of a single rhythm in the distributed active environment of the heart have been found. DR and CGR have been studied to a lesser extent in other parts of the human body. In the future, the study of the physical properties of DR, the formation of their rhythm will be investigated on the basis of the DR of the heart.

\section{Mathematical models for studying rhythm drivers}

To study the nonlinear dynamics of the formation of a unified rhythm in the general case, when the VR is a distributed active medium, a system of nonlinear partial differential equations of parabolic type is used.

$$
\frac{\partial E_{i}}{\partial t}=F_{i}(\vec{E})+D_{i} \Delta E_{i}+f_{i}(x, y, z, t) ; \quad(i=1, \ldots, n)
$$

For some cases, it is sufficient to use a system of two equations.

$$
\frac{\partial E_{1}}{\partial t}=f_{1}\left(E_{1}, E_{2}\right)+D \Delta E_{1} ; \quad \frac{\partial E_{2}}{\partial t}=f_{2}\left(E_{1}, E_{2}\right)+D \Delta E_{2}
$$

The point system for partial differential equations (1) is the system nonlinear differential equations with a small parameter at the highest derivatives [1]

$$
\varepsilon \frac{d \vec{x}}{d t}=\vec{f}\left(x, \vec{y}, \vec{\beta}_{1}(\mathrm{t})\right) ; \quad \frac{d \vec{y}}{d t}=\vec{g}\left(\vec{x}, \vec{y}, \vec{\beta}_{2}(\mathrm{t})\right)
$$

where,

$$
\begin{gathered}
\vec{x}=\left(x_{1}, \ldots, x_{k}\right), \quad \vec{y}=\left(y_{1}, \ldots, y_{l}\right), \\
\vec{f}=\left(f_{1}, \ldots, f_{k}\right), \quad \vec{g}=\left(g_{1}, \ldots, g_{l}\right), \quad \vec{\beta}_{1}(t), \vec{\beta}_{2}(t)
\end{gathered}
$$

is the synchronizing effect. The phase space of system (3) $R^{n} \quad(n=k+l)$ naturally splits into the direct sum of $k$-measured a dimensional subspace $X^{k}$ and $l$-measured a dimensional subspace $Y^{l}$. The phase picture of Eq. (3) is characterized by the presence of "fast" and "slow" motions. If, as a result of the alternation of slow and fast motions, a closed trajectory arises, then the corresponding periodic solution (3) represents a relaxation oscillation. It was found that the control of relaxation oscillations can be achieved by controlling the individual phases of the oscillations by slow or fast ones. The most effective is the control of the slow phase.

To study the sinoatrial node from a set of relaxation oscillators - pacemakers, one can use the operator equation of electrical processes in the form

$$
\vec{u}=\vec{L} \vec{u}+\vec{R}(\vec{u})
$$

The connection of the i-th pacemaker with the rest in the first approximation can be considered countably additive. If we consider the connection with the nearest neighbors to be the most important, then the equations can be used to describe the sinoatrial node

$$
u_{i}(t)=\vec{L} u_{i}(t)+\sum_{j \in\{N\}} \alpha_{i j}\left(u_{j}-u_{i}\right),(i=1, \ldots, n),
$$

where $\{N\}$ is the set of all pacemakers of the nearest environment. To build a model of competitive interaction of pacemakers in the future, we use the Fitzhue mathematical model [2]

$$
\frac{d u_{1}}{d t}=u_{1}-\frac{u_{1}^{3}}{3}-u_{2}+I, \quad \frac{d u_{2}}{d t}=\varepsilon\left(a-u_{1}+b u_{2}\right)
$$

where, I, $a, b, \varepsilon$ - parameters: $a=0.7, b=0.8$. At $I=0.14$, the system is potentially self-oscillating; at $I=$ 0.4 , the equations describe relaxation oscillations. To study competition in a distributed system, we use the Fitzhu - Nagumo mathematical model of the type of equation (2) [3]

$$
\frac{d x_{1}}{d t}=u_{1}-\frac{u_{1}^{3}}{3}-u_{2}+I+D \Delta u_{1} ; \quad \frac{d u_{2}}{d t}=\varepsilon\left(a-u_{1}+b u_{2}\right)
$$

where: $u_{1}, u_{2}$ - variables, $D$ - diffusion coefficient characterizing the propagation of autowave processes in a two-dimensional distributed active medium of pacemakers.

\section{Formation of a single rhythm of the synoatrial node of the heart. Analytical results}

There are no exact solutions to equations (3), therefore, the study of self-oscillating relaxation systems caused significant difficulties. A breakthrough in this area was 
the work of $\mathrm{N}$. Wiener and A. Rosenbluth, who formulated the axiomatic theory of active excitable media [4]. On the basis of this theory, I.M. Gel'fand proposed a hypothesis about the formation of a single rhythm in a system of relaxation oscillators with different periods of oscillations. According to this theory, in an axiomatic excitable environment, a single rhythm of self-oscillations is equal to the highest rhythm for all excitable elements [5], that is

$$
\mathrm{f}_{0}=\max _{\mathrm{k}} \mathrm{f}_{\mathrm{k}} \quad(\mathrm{k}=1, \ldots, \mathrm{n})
$$

However, the experimental results of physiologists did not confirm the Wiener-Gelfand theory [6-10]. This circumstance caused a heated debate among experimental physiologists, which is reflected in many publications. It was experimentally established that a single rhythm of the sinoatrial node is formed mainly at an intermediate level closer to the frequency of the highfrequency pacemaker.

\section{Analytical results on the formation of a single heart rhythm}

For the value of the total period $T_{0}$ of excitation in a theoretical study, the ratio was obtained [11-15]

$$
T_{0}=T_{1}+\alpha_{12} K_{2}\left(T_{2}-T_{1}\right) /\left(K_{1}+\alpha_{12}\left(K_{2}-K_{1}\right)\right)
$$

where $T_{0}$ is the period of the formed rhythm, $T_{1}, T_{2}$ are the periods of interacting pacemakers, $\alpha_{12}$ is the coupling coefficient, $K_{1}, K_{2}$ is the slope of the slow phase of the pacemakers. If $K_{1} \square K_{2}, \alpha_{12}<<1$, then, as follows from the last formula

$$
\begin{gathered}
T_{0}=T_{1}+T_{12}\left(T_{2}-T_{1}\right), \\
f_{0}=f_{\max }-\alpha_{12}\left(f_{\max }-f_{\text {min }}\right) .
\end{gathered}
$$

Thus, the results of calculations, confirmed by experimental studies, showed that the single rhythm of the pacemaker is not equal to the maximum possible in the pacemaker system, but it is less than the maximum by an amount $\alpha_{12}\left(f_{\max }-f_{\min }\right)$. N. Wiener's ingenious statement was generally confirmed with a small correction for the frequency.

For $n$ pacemaker cells, an approximate expression is obtained for $T_{0}$ in the form

$$
T_{0} \square\left(1 / k_{1}\right)\left(K_{1} T_{1}+\sum_{j=2}^{n} \alpha_{i j}\left(K_{j} T_{j}-K_{1} T_{1}\right)\right)
$$

A qualitatively approximate location of a single rhythm between the rhythms of the original pacemakers is illustrated in Fig. 2.

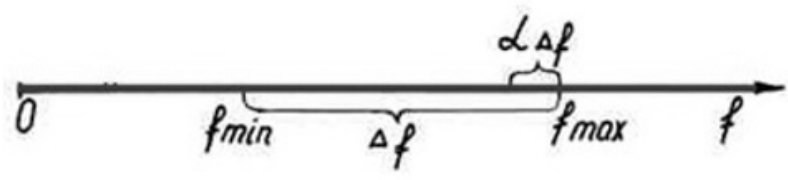

Fig. 2. The approximate location of a single rhythm between the rhythms of the original pacemakers.

\section{Basic experimental data on the formation of a single heart rhythm}

Consider the main types of experiments to study the formation of a single rhythm. Convincing results were obtained in experimental studies of the interaction in cell cultures of cardiomyocytes. The sinoatrial node was divided into separate cardiomyocyte cells using trypsin. It turned out that each isolated myocyte beats at its own rhythm. When contact is established between two myocardial cells, they synchronize after a while, that is, they begin to beat in a common rhythm. The authors believed that when synchronized, the faster cell should impose its rhythm on the slow one. However, synchronous beats usually occurred at an intermediate level between the frequencies of both pacemakers.

The study of the formation of a single rhythm of pacemaker cells was studied using the microelectrode technique during the interaction of fragments of the pacemaker tissue, and later in the interaction of single pacemaker cells. The works [7, 8] describe the interaction of individual fragments of the pacemaker tissue of the frog heart. The typical most often achieved result of interaction: the establishment of a single rhythm occurs at an intermediate level. The typical most often achieved result of interaction: the establishment of a single rhythm occurs at an intermediate level. In fig. 3, left, shows the results of one of the experiments in this series.
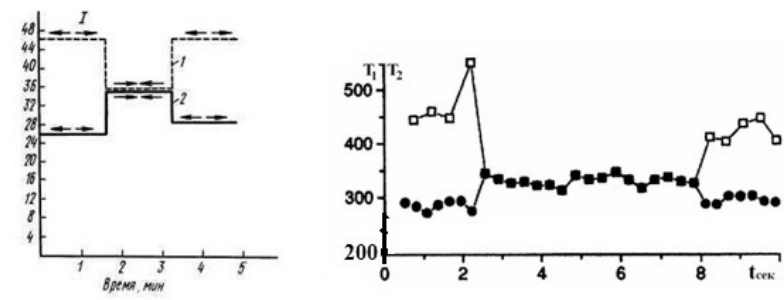

Fig. 3. Interaction of fragments of the pacemaker tissue of the frog's heart on the left; the rhythms of the preparations are 46 and 26 beats / min, the general rhythm is 35 beats / min; the right shows the interaction of two single pacemaker cells. A single rhythm is formed at an intermediate level closer to the rhythm of a cell with a higher rhythm.

The results of the initial work were brilliantly confirmed in experiments to study the interaction of two isolated pacemaker cells obtained from a rabbit tissue culture. and with computer fixation of the constancy of intercellular communication. With an increase in communication, rhythmic activity occurs in a single rhythm. These results are illustrated in fig. 3 on the right. Thus, at medium and large values of the connection, 
complete synchronization takes place, a single rhythm is formed at an intermediate level close to the level of a higher-frequency pacemaker, as shown in fig. 3 on the right. These experiments confirm the results of theoretical studies obtained in works [11-14].

\section{Rhythm drivers hierarchy}

An essential property of pacemakers developed by evolution is the formation of hierarchies. In the heart, the pacemaker is the sinoatrial node. In pathological conditions, the role of the pacemaker can be played by other underlying parts of the heart. The sinus-atrial node suppresses with more frequent impulses all the lower parts of the conduction system, but if it is damaged, the atrioventricular node, which generates impulses with a frequency of 40-50 per minute, can become a pacemaker. In the event of damage to this node, the fibers of the atrioventricular bundle (His bundle) can become a pacemaker, taking on its function. The frequency of the generated impulses and heart rate will be about 30-40 per minute. If these pacemakers do not work, then Purkinje Fibers can become them, setting the heart rate at about 20 per minute. Accordingly, the sinus node is the first order pacemaker, the atrio-gastric node is the second order pacemaker, and the Giss bundles are the third order pacemaker. Fibers of the ventricle are a fourth-order pacemaker. An illustration of the location of pacemakers of various orders in the heart is shown in fig. 4.

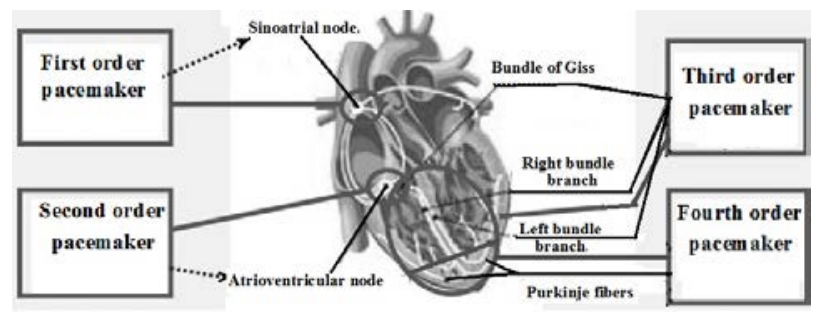

Fig. 4. Illustration of the location of pacemakers of various orders in the heart

\section{Alignment of the synoatrial node and atrials}

The DR must transmit a stable signal of sufficient electrical power to the environment. A two-dimensional model of the sinoatrial node was investigated, including pacemaker and atrial cells. The possibility of effective synchronization of atrial cells has been established. Transitional forms of cell potentials from pacemaker to atrial were found. In [15], the electrophysiological properties of the mosaic model of the sinoatrial node were investigated using a mathematical model. H. Zhang et al. a mosaic model of 20x20 dimension was investigated, including 400 cells of the sinoatrial node and atria [16]. A schematic representation of the model is shown in fig. 5 .

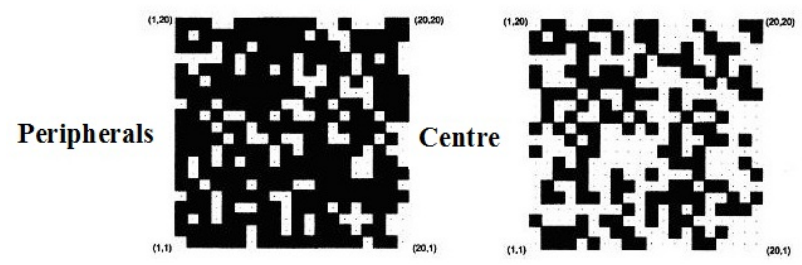

Fig. 5. Mosaic model of the center and periphery of the sinoatrial node. The lattice dimension is $20 \times 20$. The total number of nodal and atrial cells is 400 . Nodal cells are shown in white squares, atrial cells in black. a) mosaic model of the periphery, b) the central part of the node.

\section{Dynamics of competition and formation of a single rhythm of a two- dimensional synoatrial node}

A numerical experiment was performed to test the existing hypotheses. Fitzhu - Nagumo equations (5) [3] were used as a mathematical model of DR. For the numerical solution of nonlinear partial differential equations of parabolic type, which can be reduced to a system of point nonlinear differential equations, the author's "scanning method" was used, which allows finding solutions of partial differential equations in inhomogeneous domains with complex geometry of boundaries [17]. The idea of a computational experiment is schematically illustrated in Fig. 5. The interaction of 10 thousand pacemakers is considered; this number of pacemakers is characteristic of the rabbit sinoatrial node. The system is described by 20 thousand nonlinear differential equations of Van der Pol - Fitzhju. The pacemakers of the node have a rhythm of about 60 beats / min, that is, a two-dimensional self-oscillating active medium is considered. There are also 5 groups of higher frequency pacemakers: 1) a pool of 9 pacemakers with a frequency of 70 beats / min; 2) a pool of 25 pacemakers with a frequency of $65 \mathrm{bpm}$; 3) a pool of 4 pacemakers with a frequency of 70 beats / min; 4) two pacemakers with a frequency of 75 beats / min; 4) one pacemaker with a frequency of 75 beats / min; pacemakers with a frequency of 75 beats / min have the highest rhythm. The scheme of the computational experiment for studying the interaction of the pacemakers of the sinoatrial node is shown in fig. 6 on the left, on the right shows the results of a computational experiment.

In fig. 6 clearly shows that the rate of capture of the excitation region by a group of pacemakers substantially depends on the frequency of oscillations of pacemakers in the group and on the number of pacemakers in the group, that is, on the power of the group. The higher the frequency and the number of pacemakers in the group, the faster the capture of the surrounding territory. At the same time, the frequency of pacemakers has a greater impact on the rate of capture of the surrounding territory than the number of pacemakers in the group. The dynamics of the wave movement at certain moments is interrupted by the excitation of the surrounding pacemakers. This destroys the wave periphery. It can be seen that a single rhythm is formed by a group of nine pacemakers. A more numerous group of twenty-five 
pacemakers, having a lower rhythm, fails to form a common rhythm; the rhythm of a group of nine pacemakers is imposed on it.
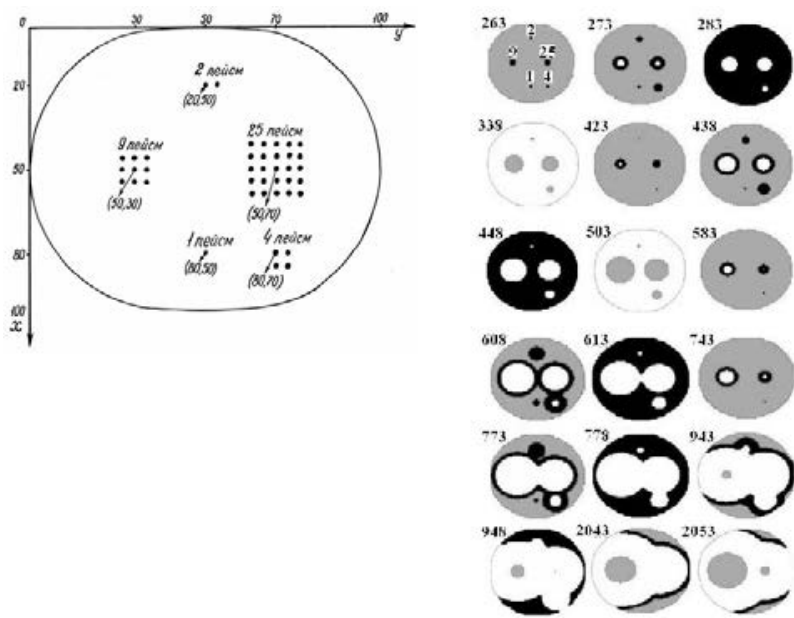

Fig. 6. The scheme of the computational experiment for studying the interaction of the pacemakers of the sinoatrial node is shown on the left, the results of the computational experiment are shown on the right.

Fig. 6 it can be seen that one pacemaker, even the highest-frequency one, cannot capture the rhythm of the entire node, nor can groups of two and four pacemakers do this, although as the number of pacemakers in a group increases, the size of their zone of influence increases. The results of a numerical experiment made it possible to visually confirm the main provisions on rhythmogenesis in the sinoatrial node of the heart: 1) rhythmogenesis is carried out by the pool with the highest rhythm; 2) the fastest cell alone cannot excite the entire node; 3) a single rhythm is established at an intermediate level close to the level of the highestfrequency cells; 4) a small number of high-frequency cells can create only a small focus of excitation (active vesicle), the size of which increases as the number of cells in the pool grows; 5) shows the efficiency of excitation of the sinoatrial node from its periphery. A further study of synchronization, competitive dynamics in neural ensembles was carried out in [18-20].

\section{Driver rate control}

Controlling the formed rhythm of DR and CGR is fundamentally different in terms of the physical mechanisms used for this. DR control is achieved due to the frequency of self-oscillations of each partial pacemaker, in connection with which the frequency of a single formed rhythm changes. In the CGR, the control of the formed rhythm is achieved by changing the parameter in the positive feedback loop. If the network is formed by oscillators, then the frequencies of these oscillators do not change.

There are constant tonic influences on the heart from the bulbar nuclei of the vagus nerve: with its bilateral transection, the frequency of palpitations increases 1.52.5 times. Stimulation of the vagus nerve causes a decrease or cessation of cardiac activity due to inhibition of the automatic function of the pacemakers of the sinoatrial node. The severity of this effect depends on the strength and frequency of stimulation of the vagus nerve. The negative chronotropic effect of irritation of the vagus nerve is associated with a slowdown in the generation of impulses in the pacemaker of the sinus node. Since, when the vagus nerve is irritated, a mediator, acetylcholine, is released at its endings, its interaction with the muscarinic receptors of the heart increases the permeability of the surface membrane of pacemaker cells for potassium ions. As a result, membrane hyperpolarization occurs, which slows down the development of slow spontaneous diastolic depolarization, and therefore the membrane potential later reaches a critical level. This leads to a decrease in the DR rhythm of the sinoatrial node. An illustration of the control of the rhythm of the sinoatrial node from the central nervous system - the medulla oblongata is shown in fig. 7.
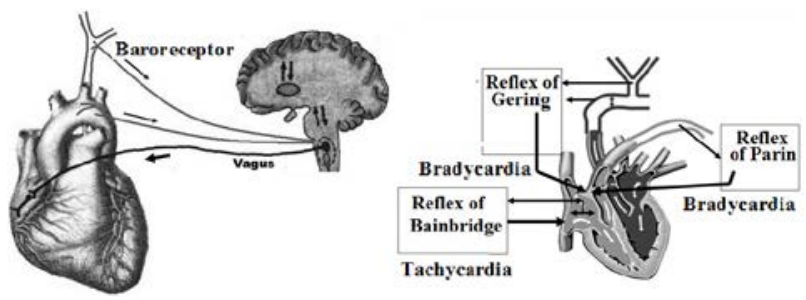

Fig. 7. Control of the rhythm of the sinoatrial node from the medulla oblongata of the central nervous system on the left, on the right, the control structure is shown using one's own cardiac reflexes. With an increase in blood pressure, the rhythm decreases. With a decrease in blood pressure, the rhythm increases.

Own cardiac reflexes form the basis of neurogenic regulation of the heart. Own reflexes of the heart are also manifested in response to mechanical stimulation of the heart chambers, in the walls of which there are a large number of baroreceptors. These include the Bainbridge reflex, which manifests itself as tachycardia in response to the rapid intravenous administration of a certain volume of blood. Among their own reflexes include the Hering reflex, which manifests itself in the form of bradycardia. The unloading reflex of V.V. Parin deserves a description: when the receptors of the pulmonary artery and pulmonary veins are irritated, hypotension is caused in the systemic circulation, and bradycardia develops. This reflex is used to unload the pulmonary circulation. Control of the rhythm of the sinoatrial node of the heart is possible by changing the duration of the phase of slow diastolic depolarization. The mechanism of this influence is described in more detail in [11-14].

\section{Conclusion}

It is shown that the pacemaker is an ensemble of many relaxation auto-generators with mutual synchronization. A mathematical model of a pacemaker in the form of a non-autonomous nonlinear system of ordinary differential equations with small parameters before derivatives is presented. For the numerical study of the 
properties of the pacemaker, a modified axiomatic Wiener-Rosenbluth method was used with the properties of uniform, almost periodic functions. It has been shown that the biological pacemaker is usually a hierarchical system. It has been shown that the human body and brain are permeated with many rhythm generators.

\section{References}

1. U.F. Mishchenko, N.Kh. Rozov, Differential equations with a small parameter and relaxation oscillations (M.: Science. 1975)

2. R.A. FitzHugh, Biophys. J., 1, 445 (1961)

3. J. Nagumo, S. Arimoto, S. Yoshizawa, Proc. IRE, 50, 2061 (1962)

4. N. Wiener, A. Rosenbluth, Conducting impulses in the heart muscle. Mathematical formulation of the problem of impulse conduction in a network of connected excitable elements, in particular in the heart muscle (M.: IL. Cybernetic. Sat., 3, 1961)

5. I.M. Gelfand, M.L. Tsetlin, DAN SSSR., 131(6), 1242-1245 (1960)

6. I.M. Gelfand, S.A. Kovalev, L.M. Chailakhyan, DAN SSSR., 148(4), 973-976 (1963)

7. Said Abu El Ela Kawser, M.G., Udelnov, G.S. Sukhova, Part II. Biological Sciences, 6, 46-49 (1979)

8. M.E. Mazurov, G.S. Sukhova, M .: NDVSH. Biological Sciences, 1(325), 140-149 (1991)

9. P.P. Aliev, L.V. Rozenshtraukh, Russian physiology. Magazine them. Sechenov, 92(9), 10691077 (2006)

10. E.E. Verheijck., R. Wilders, R. Joiner et all., J. Gen. Physiol., 111, 95-112 (1998)

11. M.E. Mazurov, Biophysics, 35(6), 494-498 (1990)

12. M.E. Mazurov, Biophysics, 35(6), 1001-1006 (1990)

13. M.E. Mazurov, Biophysics, 51(6), 1092 (2006)

14. M.E. Mazurov, ZhVM and MF AS USSR, 31(11), 1619-1636 (1991)

15. M.R. Boyett, H. Dobrzynski, M.K. Lancaster, S.A. Jones, H. Honjo, I. Kodama, J Cardiovasc Electrophysiol, 14, 104-106 (2003)

16. H. Zhang, A.V. Holden, M.R. Boyett. Circulation, 103, 584-588 (2001)

17. M.E. Mazurov, I.M. Kalyuzhny, Third International Conference "System Analysis and Information Technologies" (SAIT), 419-424 (2009)

18. M.E, Mazurov, Izvestiya RAN Phys. Series, 82(1), 83-87 (2018)

19. M.E. Mazurov, Izvestia RAN. Series nat., 82(11), 1564-1570 (2018)

20. M.E. Mazurov, Izvestia RAN. Series nat., 84(3), 451-456 (2020) 